\title{
Fowler's syndrome_progesterone deficiency or oestrogen excess?
}

Jae I. Shin

In their article, Nadir Osman and Christopher Chapple provided an excellent Review of Fowler's syndrome (Fowler's syndrome-a cause of unexplained urinary retention in young women? Nat. Rev. Urol. 11, 87-98; 2014). ${ }^{1}$ They described how a poorly relaxing external urethral sphincter might cause increased urethral afferent activity, inhibiting bladder afferent signalling and leading to poor bladder sensation and detrusor underactivity. When Fowler and colleagues ${ }^{2}$ first described this syndrome in 1988 in young women with unexplained urinary retention associated with polycystic ovary syndrome (PCOS), they speculated that a relative deficiency of progesterone, a cell-membrane stabilizer, might permit ephaptic transmission of impulses between muscle fibres in the urethral sphincter, giving rise to abnormal electromyographic activity and impairing relaxation of the sphincter.

I would like to suggest another mechanism for the pathology of this disease. PCOS is both a hyperoestrogenic and a hyperandrogenic syndrome, in which hyperoestrogenaemia is thought to be caused by an elevation of oestrone, when plasma oestradiol levels are in the normal follicular range. ${ }^{3}$ Although not reviewed by Osman and Chapple, ${ }^{1}$ evidence suggests that oestrogens could increase detrusor and urethral pressure and cause urinary retention..$^{4-7}$

Kuroda et al. ${ }^{4}$ reported that oestradiol injections resulted in urinary retention in the bladders of castrated mice, and Bump and Friedman ${ }^{5}$ showed that oestradiol replacement in hypo-oestrogenic baboons increased functional urethral length and mean urethral pressure. Streng et al. ${ }^{6}$ demonstrated that mice treated with oestradiol or oestrone showed obstructive voiding, seen as increased bladder pressure, decreased average flow-rate and prolonged micturition time. Elliott et al. ${ }^{7}$ reported that diethylstilbestrol was effective in blocking rat and human detrusor muscle contraction, possibly owing to a reduction in the influx of calcium ions into muscle cells during contraction. Moreover, Seidlová-Wuttke et al. ${ }^{8}$ showed that oestradiol increased vesicular and urethral pressure in a coordinated manner. Conversely, Flamant and Gillet ${ }^{9}$ reported that lower oestrogen levels lead to vaginal atrophy, diminished sphincter tone and increased bladder sensitivity, suggesting that oestrogens might have an important role in improving urethral trophicity.

These studies have provided evidence that supports the suggestion that hyperoestrogenaemia in PCOS might impair relaxation of the urethral sphincter, resulting in low flow-rates of urine, incomplete emptying of the bladder and, finally, urinary retention. Further studies are necessary to evaluate the relationship between the degree of hyperoestrogenaemia and detrusor or urethral pressure, and to elucidate the effect of sacral neuromodulation on the influx of calcium ions into the cell during detrusor contraction, which could be affected by oestrogens.

Department of Paediatrics, Yonsei University College of Medicine, Seoul 120 752, Republic of Korea.

shinji@yuhs.ac

Competing interests

The author declares no competing interests.

1. Osman, N. I. \& Chapple, C. R. Fowler's syndrome - a cause of unexplained urinary retention in young women? Nat. Rev. Urol. 11, 87-98 (2014)

2. Fowler, C. J. et al. Abnormal electromyographic activity of the urethral sphincter, voiding dysfunction, and polycystic ovaries: a new syndrome? BMJ 297, 1436-1438 (1988).

3. Benjamin, F. et al. Excessive estradiol secretion in polycystic ovarian disease. Am. J. Obstet. Gynecol. 169, 1223-1226 (1993).

4. Kuroda, H. et al. Urinary retention induced by estrogen injections in mice: an analytical model. J. Urol. 134, 1268-1270 (1985).

5. Bump, R. C. \& Friedman, C. I. Intraluminal urethral pressure measurements in the female baboon: effects of hormonal manipulation. J. Urol. 136, 508-511 (1986).

6. Streng, T. K. et al. A dose-dependent dual effect of oestrogen on voiding in the male mouse? BJU Int. 96, 1126-1130 (2005).

7. Elliott, R. A. et al. The direct effects of diethylstilboestrol and nifedipine on the contractile responses of isolated human and rat detrusor muscles. Eur. J. Clin. Pharmacol. 43, 149-155 (1992).

8. Seidlová-Wuttke, D. et al. Urodynamic effects of estradiol (E2) in ovariectomized (ovx) rats. Endocrine 23, 25-32 (2004).

9. Flamant, G. \& Gillet, J. Y. Drug therapy of female urinary incontinence. Presse Med. 24, 31-34 (1995). 